\title{
Optimum folding pathways of proteins: Their determination and properties
}

Uğur Güner, Yaman Arkun, and Burak Erman

Citation: The Journal of Chemical Physics 124, 134911 (2006); doi: 10.1063/1.2181976

View online: http://dx.doi.org/10.1063/1.2181976

View Table of Contents: http://aip.scitation.org/toc/jcp/124/13

Published by the American Institute of Physics

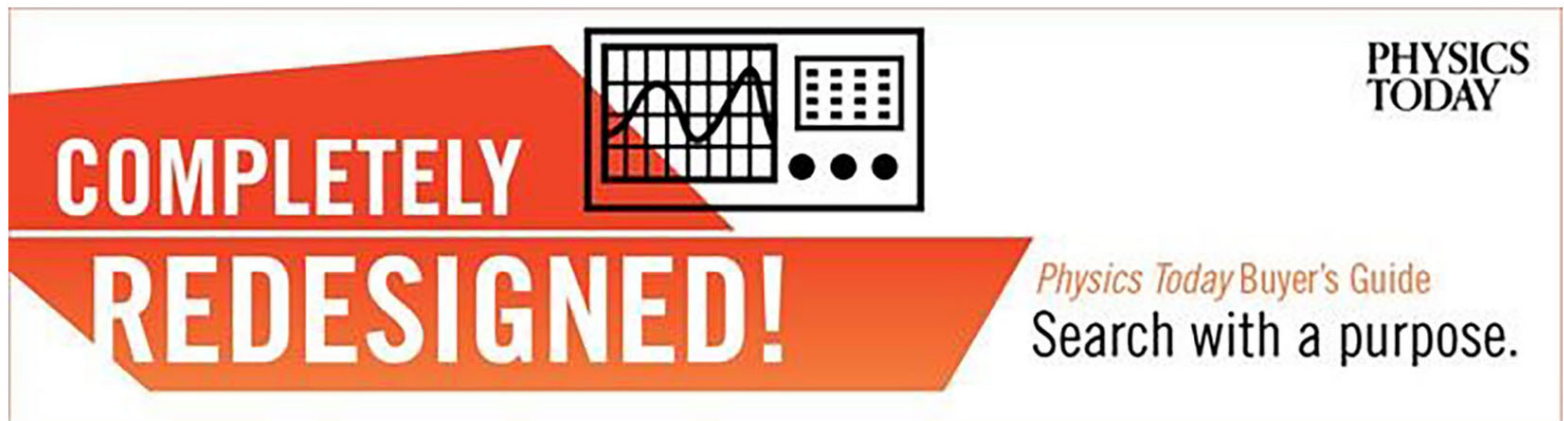




\title{
Optimum folding pathways of proteins: Their determination and properties
}

\author{
Uğur Güner, Yaman Arkun, and Burak Erman ${ }^{\text {a) }}$ \\ College of Engineering, Koc University, Rumeli Feneri Yolu, 34450 Sarlyer, Istanbul, Turkey
}

(Received 14 December 2005; accepted 8 February 2006; published online 7 April 2006)

\begin{abstract}
We develop a dynamic optimization technique for determining optimum folding pathways of proteins starting from different initial configurations. A coarse-grained Go model is used. Forces acting on each bead are (i) the friction force, (ii) forces from bond length constraints, (iii) excluded volume constraints, and (iv) attractive forces between residue pairs that are in contact in the native state. An objective function is defined as the total attractive energy between nonbonded residues, which are neighbors in the native state. The objective function is minimized over all feasible paths, satisfying bond length and excluded volume constraints. The optimization problem is nonconvex and contains a large number of constraints. An augmented Lagrangian method with a penalty barrier function was used to solve the problem. The method is applied to a 36-residue protein, chicken villin headpiece. Sequences of events during folding of the protein are determined for various pathways and analyzed. The relative time scales are compared and scaled according to experimentally measured events. Formation times of the helices, turn, and the loop agree with experimental data. We obtain the overall folding time of the protein in the range of $600 \mathrm{~ns}-1.2 \mu \mathrm{s}$ that is smaller than the experimental result of 4-5 $\mu$ s, showing that the optimal folding times that we obtain may be possible lower bounds. Time dependent variables during folding and energies associated with shortand long-range interactions between secondary structures are analyzed in modal space using Karhunen-Loeve expansion. (c) 2006 American Institute of Physics.

[DOI: $10.1063 / 1.2181976]$
\end{abstract}

\section{INTRODUCTION}

Protein folding is the process by which a protein assumes its functional shape or conformation. Incorrectly folded proteins resulting from errors during folding are responsible for illnesses such as Creutzfeldt-Jakob disease, Bovine spongiform encephalopathy, and Parkinson's and Alzheimer's diseases. Because of its relationships to these diseases, the dynamics of folding has received major attention in recent years.

The entire duration of the folding process varies dramatically depending on the protein of interest. The slowest folding proteins require many minutes or hours to fold. However, small proteins, with lengths of a hundred or so amino acids, typically fold on time scales of milliseconds or even microseconds. ${ }^{1,2}$ Power laws between the number of residues and folding times are well elucidated in recent work. ${ }^{1,3}$

The all-atom visualization of a protein coupled with molecular dynamics simulations gives the most detailed picture of folding but the computational time is a serious bottleneck. The only full-trajectory molecular dynamics simulation in the presence of explicit water up to this date is that of a 36-residue protein. ${ }^{4}$ Various coarse-graining techniques are used to override the computational time bottleneck. In the simplest coarse-grained approximation ${ }^{5,6}$ the protein consists of $N$ beads joined into a linear chain by virtual bonds analogous to the chemical bond. Each bead represents an amino acid residue that may be one of the 20 possible types, each of

\footnotetext{
${ }^{\text {a) }}$ Author to whom correspondence should be addressed. Electronic mail: berman@ku.edu.tr
}

which consists of several atoms. The system of skeletal bonds between consecutive $\alpha$-carbons is coplanar, and the intervening amide bond is in the trans state due to its partial double bond character. ${ }^{7}$ Therefore, the length of each virtual bond connecting consecutive $\alpha$-carbon atoms is fixed. Each bead has a finite volume. No bead shares its own volume with any other bead. This is called the "excluded volume condition." Folding of the protein progresses from a random initial state at $t=0$ to the final state at $t=t_{f}$, subject to the fixed bond length and excluded volume conditions at all stages of folding. The "native configuration" of the protein is a unique final configuration to which it folds irrespective of its initial configuration and is determined by the primary structure.

Folding is a competition between attractive and repulsive forces. Attractive ones are those that tend to bring two residues near each other. The hydrophobic force is an example. Hydrophobic residues tend to escape the aqueous environment of the cell and cluster at the inner regions of the protein chain, leaving the exterior to hydrophilic residues. These are nonspecific forces and they operate on all hydrophobic residues. There are also specific attractive forces between certain residue pairs that are mostly effective in giving the final shape of the protein. Repulsive forces are the excluded volume forces. In the absence of repulsive forces, the beads move along the negative gradient of the energy. Excluded volume effects, combined with bond length constraints, complicate the folding process. The complexity of the protein folding problem is similar, in many respects, to the emergence of street patterns in cities. ${ }^{8}$ In the latter, trav- 
elers want to go to a common destination in the shortest possible time along straight paths, but there are constraints imposed by property that cannot be trespassed. As a result, travelers move along a network of streets whose configurations optimize the travel time subject to the constraints imposed by the streets themselves. Temporal and spatial organization in nature obeys similar patterns: there is an objective to be optimized and there are constraints that make the optimization more and more difficult. In this sense, the protein folding problem in its simplest form may be viewed as a constrained optimization problem:

Optimization formulation of protein folding. We are given an initial configuration of $N$ beads connected in the form of a linear chain. The beads want to travel to their specified final destinations by spending minimal energy subject to the (i) connectivity between beads, (ii) bond length, and (iii) excluded volume constraints. Each bead obeys Newton's second law of motion throughout the folding trajectory. The forces acting on each bead are received either from the other beads or they are external interaction forces with the environment. Under these conditions, determine the optimal forces acting on the beads and the resulting optimal trajectory of the beads from their initial configuration to their final native states.

This formulation contains no empirical elements and the problem is purely geometric. Additional information are needed, however, to solve a given folding problem. These information come from empirical data. An objective to be minimized (or system energy function) must be specified first. The important part of the energy function is the attractive part. The total attractive energy in the system may be written as the sum of pairwise components. Lennard-Jones or the more general Mie potentials are examples. ${ }^{9}$ Second, the native state has to be defined explicitly. A widely used practice is to define the contact map. Two residues are said to be in contact if they are within $7 \AA$ of each other. ${ }^{10}$ The definition of the energy should ensure that the contact map of the native protein would be obtained at the end of folding. Variational approaches for extracting potentials that lead to the native shape are well studied. ${ }^{11}$ The folding problem as stated here is obviously a highly simplified picture of the real phenomenon. In the real case, the empirical energy functions are much more detailed and are optimized over a large set of proteins using all-atom calculations. ${ }^{12}$

The specific aim of the present study is to develop an optimization technique for solving the simplest optimization problem of protein folding stated above. We do this by employing optimal control framework in the following sections. It is not known whether a real protein chooses the optimum path while folding from a given initial to its native state. If it does, then it must be the result of evolution, which is beyond the intent of the present study. Here, we are more interested in presenting a method that can easily generate optimum paths, analyzing the sequences of events along optimized trajectories of simple protein models, and investigating the dynamic patterns that result from such optimized trajectories. Efforts in searching for such optimized trajectories may seem superfluous. However, with advances in spectroscopic techniques the knowledge of an "optimum folding trajectory" would be invaluable to synthesize a desired protein folding by manipulation at the molecular scale. Mutations caused in the laboratory are also widely used in changing the folding pathways. Chaperones are natural control mechanisms. Different control techniques that optimize protein folding have been addressed both experimentally and theoretically. ${ }^{13}$

The organization of the paper is as follows: In Sec. II, we introduce the model and the mathematical formulation of the optimization problem of protein folding. In Sec. III, we analyze the folding of the widely studied villin headpiece, which is a protein of 36 residues. Analysis of several optimized folding trajectories of the villin headpiece gives information on the main features of the folding process that is presented in detail in Sec. IV. We discuss the results of analysis in Sec. 5.

\section{THE MODEL}

We use the coarse-grained model where we represent the amino acid residues with spherical beads centered at the $\mathrm{C}^{\alpha}$ atoms. Distances between bonded pairs, i.e., the virtual backbone bonds, are adopted as $3.8 \AA .^{14}$

We divide the total energy of the system into energy arising from the interactions of bonded and nonbonded beads, $E^{B}$ and $E^{\mathrm{NB}}$, respectively. Each of these is separated into an attractive and a repulsive component. Thus for a system with $N$ beads,

$$
E=\sum_{i=1}^{N-1}\left(E_{i, i+1 ; A}^{B}+E_{i, i+1 ; R}^{B}\right)+\sum_{i>j+1}^{N}\left(E_{i j, A}^{\mathrm{NB}}+E_{i j, R}^{\mathrm{NB}}\right) .
$$

Here, subscripts $i$ and $j$ denote the residue indices, $A$ and $R$ denote the attractive and repulsive components, and the superscripts $B$ and NB denote the bonded and nonbonded components, respectively.

The equation of motion follows from Newton's second law as

$$
m \frac{d^{2} r_{i}}{d t^{2}}=-\gamma \frac{d r_{i}}{d t}+f_{i} \text { for } i=1,2, \ldots, N
$$

Here, the vector $r_{i}$ represents the position of the $i$ th bead with respect to a fixed frame coordinate, $m$ is the mass of the residue, $\gamma$ is the friction coefficient with dimensions of (force) (time)/(distance), and $f_{i}$ stands for the forces acting on the $i$ th residue. It is to be noted that Eq. (2) is a deterministic equation and no random forces act on the particles. Therefore, a given initial configuration always leads to the same folding pathway. If Eq. (2) were the Langevin equation that contained random stochastic forces, then the folding pathways (starting from the same initial configurations) should show some variation.

Conforming with the different components of energy [see Eq. (1)], we express the force term $f_{i}$ in terms of its components as well:

$$
f_{i}=f_{i, A}^{B}+f_{i, R}^{B}+f_{i, A}^{\mathrm{NB}}+f_{i, R}^{\mathrm{NB}} .
$$

Attractive forces between bonded residues $f_{i, A}^{B}$ are treated as linear spring forces. The equations for these attractive forces can be derived by assuming an empirical expression such as 
the Mie potential ${ }^{9}$ for the attractive bonded component of energy:

$$
E_{A}^{\mathrm{NB}}=\frac{1}{2} \sum_{i, i+1}^{N-1} a_{i, i+1}\left\|r_{i+1}-r_{i}\right\|^{2},
$$

where $r_{i, i+1}=\left\|r_{i}-r_{i+1}\right\|$ is the distance between the $i$ th and $i$ +1 st residues. Assuming that $a_{i, i+1}=-a$ for all $i$ without any loss of generality, one can express Eq. (4) in a compact quadratic form

$$
E_{A}^{B}=\frac{1}{2} r^{T} \Gamma_{A}^{B} r
$$

where $r=\left[x_{1}, x_{2}, \ldots, x_{N}, y_{1}, y_{2}, \ldots, y_{N}, z_{1}, z_{2}, \ldots, z_{N}\right]^{T}$ is the system position vector made up of the individual residue position vectors. $\Gamma_{A}^{B}$ is the linear connectivity matrix. ${ }^{15}$ It is a symmetric Toeplitz matrix with the first off-diagonal elements equal to -1 and the diagonal elements equal to the negative sum of the corresponding row without its diagonal element.

Next, we collect all the attractive bonded forces acting on all the beads under a single force vector $f_{A}^{B}$ :

$$
f_{A}^{B}=\left[\begin{array}{l}
f_{1, A}^{B} \\
f_{2, A}^{B} \\
\vdots \\
f_{N, A}^{B}
\end{array}\right]=\left[\begin{array}{l}
-\nabla_{r_{1}} E_{A}^{B} \\
-\nabla_{r_{1}} E_{A}^{B} \\
\vdots \\
-\nabla_{r_{N}} E_{A}^{B}
\end{array}\right]=-\nabla_{r} E_{A}^{B}=-\Gamma_{A}^{B} r .
$$

We lump the remaining force terms in Eq. (3) (i.e., bonded repulsive, nonbonded attractive, and nonbonded repulsive ones) and call it $u_{i}$ :

$$
u_{i}=f_{i, R}^{B}+f_{i, A}^{\mathrm{NB}}+f_{i, R}^{\mathrm{NB}} .
$$

Collecting all these forces under a single vector one gets

$$
u=\left[\begin{array}{l}
u_{1} \\
u_{2} \\
\vdots \\
u_{N}
\end{array}\right] .
$$

Since mass times acceleration terms are several orders of magnitude smaller than friction and intermolecular forces, the left hand side of equation of motion, Eq. (2) may be equated to zero. Also without any loss of generality, we let the friction coefficient to equate to unity.

With these definitions and assumptions, the equation of motion simplifies to

$$
\frac{d r}{d t}=-\Gamma_{A}^{B} r+u
$$

This model consists of $N$ differential equations and is expressed in classical state-space form where $r(t) \in R^{N}$ is the state and $u(t) \in R^{N}$ is the input. It is important to clarify and stress the role of the force vector $u(t)$. These forces are nonlinear forces that represent the repulsive forces between all the beads and the attractive forces that may exist among the nonbonded beads. They define the force field that helps to fold a protein from an initial condition to a final native state in a feasible (i.e., without violating excluded volume and bond length constraints) and an optimal way (i.e., with re- spect to the objective function that will be defined later). In this context, it is appropriate to interpret them as control inputs steering each bead to achieve the desired folding. In our optimal control approach, which will be discussed in the next section, these control inputs are optimally computed. At equilibrium, the left hand side of Eq. (9) vanishes, leading to the equilibrium values $u_{\text {eq }}$ in terms of the equilibrium positions $r_{\mathrm{eq}}$ of the beads as

$$
u_{\mathrm{eq}}=\Gamma_{A}^{B} r_{\mathrm{eq}}
$$

Inasmuch as $\Gamma_{A}^{B}$ is the connectivity matrix, the $i$ th force $u_{\mathrm{eq}}$ acting on the $i$ th bead is in the plane of the bonds $i-1$ and $i$.

It is to be noted that the present model assumes a known native contact map and is therefore a Go model.

\section{THE OPTIMIZATION FORMULATION}

Our optimization problem focuses on native contact pairs and tries to bring them to their native state separations during the folding process. This is accomplished by minimizing the attractive energies between the nonbonded native contact pairs. Native contact pairs are the pairs that are two or more residues apart and separated by less than $7 \AA$ in the native state and are defined by the contact map.

Since we choose the energy of the attractive forces between nonbonded pairs as the quantity to minimize, further elaboration on this point is needed. In all-atom molecular dynamics simulations in water, the attractive forces between two atoms result mainly from the attractive part of the Lennard-Jones potentials and the hydrophobic potential. In the present coarse-grained approximation, the attractive forces between pairs of beads that are neighbors in the native state, defined by the contact map, may be considered as an approximation to the real picture.

We represent the attractive energy $E_{A}^{\mathrm{NB}}$ of nonbonded native contact pairs as

$$
E_{A}^{\mathrm{NB}}=\frac{1}{2} \sum_{i>j+1} b_{i j}\left\|r_{i}-r_{j}\right\|^{2},
$$

where $b_{i j}$ are constants and $r_{i j}=\left\|r_{i}-r_{j}\right\|$ is the distance between the $i$ th and $j$ th native contact pairs. Assuming that $b_{i j}$ 's are equal to unity without any loss of generality, one can express Eq. (11) in quadratic form

$$
E_{A}^{\mathrm{NB}}=\frac{1}{2} r^{T} Q r
$$

where $Q$ is the contact matrix where the $i j$ th element is equal to -1 if the $i$ th and $j$ th residues are in contact with the native state and zero otherwise. The diagonal terms equate to the negative sum of the corresponding row without its diagonal element.

We can now state the optimization problem as a constrained optimal control problem ${ }^{16}$ that performs the following minimization for the time period between initial time $t$ $=0$ and final time $t_{f}$ : 
Objective:

$$
\underset{u(t)}{\operatorname{Min}}\left[\int_{0}^{t_{f}} E_{A}^{\mathrm{NB}}(t) d t=\frac{1}{2} \int_{0}^{t_{f}} r^{T}(t) Q r(t) d t\right] .
$$

The states $r(t)$ have to satisfy the equation of motion or the following state-space model:

$$
\begin{aligned}
& \dot{r}=-\Gamma_{A}^{B} r+u(t), \\
& r(t=0)=r_{0} \quad \text { (initial condition). }
\end{aligned}
$$

For the optimal solution to be feasible bond length and excluded volume constraints must also be included in the optimization formulation.
It is straightforward to show that the square of the bond length between $i$ th and $i+1$ th beads is given by

$$
\left\|r_{i, i+1}-r_{i}\right\|^{2}=l_{i, i+1}^{2}=r^{T} H_{i, i+1} r,
$$

where $\quad r=\left[x_{1}, x_{2}, \ldots, x_{N}, y_{1}, y_{2}, \ldots, y_{N}, z_{1}, z_{2}, \ldots, z_{N}\right]^{T}$ and $H_{i, i+1}=C_{i, i+1}^{T} C_{i, i+1}$. For a system of $N$ beads in three dimensions $C_{i, i+1}$ is a $3 \times 3 N$ matrix that has 1 and -1 in the adjacent entries in each of its three rows. For example, when $N$ $=5$ for the bond length between the first and second beads $C_{1,2}$ is given by

$$
C_{1,2}=\left[\begin{array}{ccccccccccccccc}
1 & -1 & 0 & 0 & 0 & 0 & 0 & 0 & 0 & 0 & 0 & 0 & 0 & 0 & 0 \\
0 & 0 & 0 & 0 & 0 & 1 & -1 & 0 & 0 & 0 & 0 & 0 & 0 & 0 & 0 \\
0 & 0 & 0 & 0 & 0 & 0 & 0 & 0 & 0 & 0 & 1 & -1 & 0 & 0 & 0
\end{array}\right] .
$$

Thus, with a $\pm 10 \%$ tolerance, for example, the bond length constraint is included in the optimization as follows:

$$
0.9 l_{b}^{2} \leqslant l_{i, i+1}^{2} \leqslant 1.1 l_{b}^{2} \text { for all } i=1,2, \ldots, N-1,
$$

where $l_{b}$ is the bond length. In the above formulation bond length constraints are relaxed and converted to inequality constraints [Eq. (17)] which are easier to handle by the optimization. The bond length distance $l_{b}$ is taken as $3.8 \AA$.

Excluded volume constraints are treated in a similar fashion by expressing them in terms of the state or position vector $r(t)$. Let ev $\mathrm{v}_{i j}$ denote the square of the excluded volume distance between $i$ th and $j$ th beads. Then the following holds:

$$
\mathrm{ev}_{i j}=\left\|r_{i}-r_{j}\right\|^{2}=r^{T} L_{i, j} r
$$

and $L_{i, j}=D_{i, j}^{T} D_{i, j}$. For a system of $N$ beads in three dimensions $D_{i, j}$ is a $3 \times 3 N$ matrix that has 1 and -1 in the nonadjacent entries in each of its three rows. For example, when $N=5$ for the excluded volume distance between the first and third beads $D_{1,3}$ is given by

$$
D_{1,3}=\left[\begin{array}{ccccccccccccccc}
1 & 0 & -1 & 0 & 0 & 0 & 0 & 0 & 0 & 0 & 0 & 0 & 0 & 0 & 0 \\
0 & 0 & 0 & 0 & 0 & 1 & 0 & -1 & 0 & 0 & 0 & 0 & 0 & 0 & 0 \\
0 & 0 & 0 & 0 & 0 & 0 & 0 & 0 & 0 & 0 & 1 & 0 & -1 & 0 & 0
\end{array}\right] .
$$

Excluded volume constraints are included in the optimization as follows:

$$
\mathrm{ev}_{i j} \geqslant d_{i j},
$$

where $d_{i j}$ are the square of the known excluded volume distances. For the native contact pairs, $d_{i j}$ depends on the type of residue pairs. These distances are set to the known values of the native state. The excluded volume distance between non-native pairs is set to the value of $5.1 \AA$. This value is the approximate hydrogen bond length.

Finally the magnitude of the forces is bounded to keep them realistic. The maximum and minimum limits on the forces are set equal to 2 and -2 , respectively. If these limits are chosen smaller, optimization is not able to find a feasible solution (i.e., folding to native state is not possible). For the higher limits, the changes in the states are very abrupt due to overly aggressive forces. When the limit is taken as 2 , we obtain smooth state trajectories and observe more realistic folding patterns with good resolution. Therefore, the final constraint is given by

$$
-2 \leqslant u_{i}(t) \leqslant 2 .
$$

The above optimization problem is first converted to a nonlinear program (NLP) by discretizing the state $x(t)$ and control trajectories $u(t)$ over finite elements by using polynomials. In this work the method presented by Biegler et al. ${ }^{17}$ was used. 
The time variable in our model is divided into ne intervals $\left(t_{0}<t_{1}<\cdots<t_{n e}=t_{f}\right)$. The state variable or the position vector $r(t)$ is expressed by a monomial basis expansion on each interval as

$$
r(t)=r_{i-1}+h_{i} \sum_{q=1}^{\text {ncol }} \Omega_{q}\left(\frac{t-t_{i-1}}{h_{i}}\right) \frac{d r}{d t_{i, q}},
$$

where $h_{i}$ is the length of the finite element, ncol is the number of collocation points used on the finite element $i, r_{i-1}$ is the value of the state variable at the beginning of the element $i, d r / d t_{i, q}$ is the value of its first derivative in the element $i$ at the collocation point $q$, and $\Omega_{q}$ is the polynomial of order ncol. Inside each finite element, differential state equations are satisfied at the collocation points. We used two collocation points (i.e., ncol=2) and these points were chosen to be Radau points since the end of each element is a collocation point so that constraints can be defined at the end of each element. Radau points are also known to enhance stability. ${ }^{18}$ $\Omega_{q}$ is chosen as the Lagrange function. In addition, each bond length $l_{k}$ and excluded volume $\mathrm{ev}_{i j}$ are expressed as

$$
\begin{aligned}
& l_{i, i+1}^{2}(t)=\sum_{q=1}^{\text {ncol }} \Psi_{q}\left(\frac{t-t_{i-1}}{h_{i}}\right) l_{(i, i+1)_{i, q}}^{2}, \\
& \operatorname{ev}_{i j}(t)=\sum_{q=1}^{\text {ncol }} \Psi_{q}\left(\frac{t-t_{i-1}}{h_{i}}\right) \operatorname{ev}_{(i j)_{i, q}} .
\end{aligned}
$$

The control profile is similarly discretized by

$$
u(t)=\sum_{q=1}^{\text {ncol }} \Psi_{q}\left(\frac{t-t_{i-1}}{h_{i}}\right) u_{i, q},
$$

where $\Psi_{q}$ are Lagrange polynomials.

Substitution of Eqs. (22)-(25) into the original optimization problem Eqs. (13)-(21) one obtains the following NLP in which the original variables and equations are expressed in terms of the new variables $x(t)$ which are defined at the collocation points of the finite elements:

$$
\operatorname{Min}_{x \in R^{n}} f(x)
$$

subject to

$$
\begin{aligned}
& g_{i}(x) \leqslant 0, \quad i=1, \ldots, m_{g}, \\
& h_{i}(x)=0, \quad i=1, \ldots, m_{h},
\end{aligned}
$$

where $x=\left[d r / d t_{i, q}, r_{i}, \mathrm{ev}_{(i j)_{i, q}}, l_{(i, i+1)_{i, q}}^{2}, u_{i, q}\right]^{T}$ and $f, g_{i}$, and $h_{i}$ are functions from $R^{n}$ to $R$ and $m_{g}$ and $m_{k}$ are the number of inequality and equality constraints. Note that inequality constraints $g_{i}$ are the bond length and excluded volume constraints, equality constraints $h_{i}$ are the state equations of motion, and $f$ is the objective function, all expressed in terms of the new variables $x(t)$.

The above NLP is nonconvex and it contains a large number of constraints, as shown in the example below. The resulting NLP problem is next solved by a penalty barrier method $^{19}$ using the solver PENNON. ${ }^{20}$ PENNON is originally developed for convex semi-definite programming problems. ${ }^{19}$ It has also been generalized and tested on nonconvex, nonlinear problems with success. ${ }^{20}$ The optimization problem was written in the AMPL environment.

The optimization adjusts the forces $u(t)$ to bring the native contact pairs to their native state separation as close as possible. The dynamic model given by Eq. (14) guarantees the connectivity between beads and governs the motion of the beads under the force field that is adjusted by the optimization. Optimization computes the optimal force field $u(t)$ and the optimal state trajectory $r(t)$ over the time interval $\left(0, t_{f}\right)$. Optimization tries to satisfy the excluded volume and bond length constraints while bringing the native contact pairs together, thus consequently folding the protein. We choose the final time long enough to let the chain settle to a final position and short enough to decrease the optimization running time. It is important to recognize that this optimization offers a flexible framework to study the folding process in general. It offers a useful way to generate optimal trajectories to the native state. This is important if we consider the difficulty of avoiding many physical constraints that shape the folding pathways, let alone optimality. Second, different types of objective functions may be used to generate and study alternative folding pathways.

\section{MODAL ANALYSIS OF THE FORCE FIELD}

The contribution of the different modes of the force field to the minimization of the total energy gives important information of the folding process. Each force acting on a bead has its $x, y$, and $z$ components in three dimensions, $u_{i}(x)$, $u_{i}(y)$, and $u_{i}(z)$, respectively. Since there are 36 beads in total, the optimization algorithm computes $(36 \times 3=108)$ force components for each time step $t_{i}$ of the folding process. Thus, we can construct the following force matrix $U$ which contains the individual forces acting on all 36 beads:

$$
U=\left[u\left(t_{1}\right) \cdots u\left(t_{M}\right)\right]
$$

where $M$ is the number of time steps which is 301 in our simulations. Therefore, $U$ is a $108 \times 301$ matrix. The dominant dynamics or features of this force field can be studied by performing a particular principal component analysis known as Karhunen-Loeve expansion ${ }^{6}$ (KLE) on the above force matrix $U$. We can summarize the application of KL expansion to our data briefly as follows.

Given the force matrix $U$ [Eq. (28)] first construct its covariance matrix $\Phi$ :

$$
\Phi=\frac{1}{M} U U^{T}
$$

Next perform the eigenvalue decomposition of $\Phi$ :

$$
\Phi \phi_{j}=\lambda_{j} \phi_{j}
$$

where $\lambda_{j}$ is the $j$ th eigenvalue and $\phi_{j}$ is the $j$ th eigenvector of $\Phi$. The original force matrix can be reconstructed by its 
TABLE I. Secondary structures and their corresponding residue numbers.

\begin{tabular}{cc}
\hline \hline Residue No. & Secondary structure \\
\hline $4-8$ & Helix 1 \\
$9-14$ & Loop \\
$15-18$ & Helix 2 \\
$19-22$ & Turn \\
$23-30$ & Helix 3 \\
\hline \hline
\end{tabular}

KL expansion:

$$
U\left(t_{i}\right)=\sum_{j=1}^{N} c_{j}\left(t_{i}\right) \phi_{j} \quad \text { for } i=1,2, \ldots, M .
$$

In this equation, $c_{j}$ stands for the time varying amplitude of the $j$ th eigenvector, and it is called the temporal mode or modal amplitude. It can be calculated from

$$
c_{j}\left(t_{m}\right)=u^{T}\left(t_{m}\right) \phi_{j},
$$

where the superscript $T$ in Eq. (32) denotes the transpose. The KL expansion expressed by Eq. (31) has $N$ modes. If the $\mathrm{KL}$ expansion retains only the first $L$ modes (instead of all the $N$ modes), only the first $L$ important trends are extracted and the less significant details (i.e., higher modes) are filtered.

\section{APPLICATION: OPTIMUM FOLDING TRAJECTORIES OF THE PROTEIN CHICKEN VILLIN HEADPIECE}

In our study, we applied our method to a 36-residue protein (the PDB code 1vii.pdb), chicken villin headpiece that is the smallest protein that can fold autonomously. Following the work of Duan and Kollman ${ }^{4}$ the folding dynamics of this protein has been widely studied by several independent groups. ${ }^{21-27}$ Chicken villin headpiece has three short helices. We refer to them as helices 1,2 , and 3. These helices contain the residues $4-8,15-18$, and $23-30$, respectively. They are held together by a loop between residues 9-14 and a turn between residues 19-22 (see Table I).

Starting from 19 random initial configurations the optimal folding trajectories were calculated for further analysis. The results are discussed in the sequel.

\section{A. Behavior of short- and long-range contact pairs}

We can separate the native contact pairs into two groups: short-range and long-range pairs. Native contact pairs are the pairs that are two or more residues apart and separated by less than $7 \AA$ in the native state. The long-range pairs are more than five residues apart. Short-range pairs are five or less residues apart. In our optimization problem, there are 89 native contacts; 8 of them are long-range and 81 of them are short-range contacts. Native contacts are listed in Table II.

TABLE II. Long- and short-range contact pairs.

\begin{tabular}{ll}
\hline \hline Short-range & All pairs $i, i+k k=2-5$ \\
contact pairs & $\left\|r_{i, i+k}\right\|<7.0 \AA$ \\
Long-range & $(2,34)(7,14)(7,34)(10,33)$ \\
contact pairs & $(10,34)(11,33)(11,34)(19,26)$ \\
\hline
\end{tabular}

TABLE III. Final rmsd values of substructures and whole chain for the optimization cases, with all native contacts and with only short-range native contacts.

\begin{tabular}{lcc}
\hline \hline & $\begin{array}{l}\text { Final rmsd values }(\AA) \text { for } \\
\text { the optimization with only } \\
\text { short-range native contacts }\end{array}$ & $\begin{array}{l}\text { Final rmsd values }(\AA) \text { for } \\
\text { the optimization with all } \\
\text { native contacts }\end{array}$ \\
\hline Helix 1 & 0.090 & 0.0638 \\
Loop & 1.140 & 0.0999 \\
Helix 2 & 0.100 & 0.0656 \\
Turn & 0.741 & 0.1190 \\
Helix 3 & 0.060 & 0.0595 \\
The chain & 10.86 & 0.8258 \\
\hline \hline
\end{tabular}

Our analysis uses the root mean square deviation (rmsd) that compares the structure of a segment between residues $n_{1}$ and $n_{2}$ according to

$$
\operatorname{rmsd}=\left(\sum_{i=n_{1}}^{n_{2}}\left(r_{i}-r_{i}^{0}\right)^{2}\right)^{1 / 2} /\left(n_{2}-n_{1}+1\right) .
$$

Here, $r_{i}^{0}$ represents the position vector of the $i$ th residue of the native state. The latter is the reference state taken as the PDB structure of the protein.

Table III shows that when all native contacts (short and long range) are included in the objective function, optimization reduces the rmsd for the whole chain to very small values, below $1 \AA$. On the other hand, if we omit the long-range contacts in the objective function, the resulting rmsd value is an order of magnitude higher for the chain.

Figure 1(a) shows a representative final configuration obtained in the absence of long-range forces. It is seen that secondary structures form in the absence of long-range forces, indicating that long-range forces are not necessary to stabilize them. This is in contrast to earlier observations where Mori et al. ${ }^{25}$ observed that the helices are not stable by themselves and their stability relies on long-range interactions. Their work agrees with the work of Tang et al. who showed through NMR and circular dichroism (CD) measurements that the helices are stable only after a significant amount of long range contacts are established. ${ }^{22}$ We should note that the Go-like potentials adopted here do not discriminate between amino acid specific interactions, and this may explain the discrepancy between our results and those from Mori et al. $^{25}$ or experiments. ${ }^{22,23}$ The final configuration obtained in the presence of both long- and short-range forces is shown in Fig. 1(b). The native configuration is shown in Fig. 1(c).

Another important observation is that when the longrange attractive forces are absent the time for the rmsd values (a)

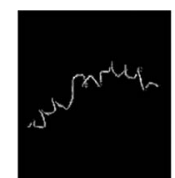

(b)

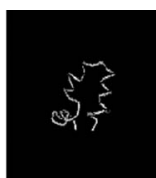

(c)

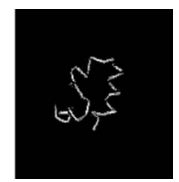

FIG. 1. Final configurations. (a) The final configuration reached by our model when long-range contacts are omitted. (b) Final configuration reached by our model when all native contacts are included. (c) Native state of protein. 


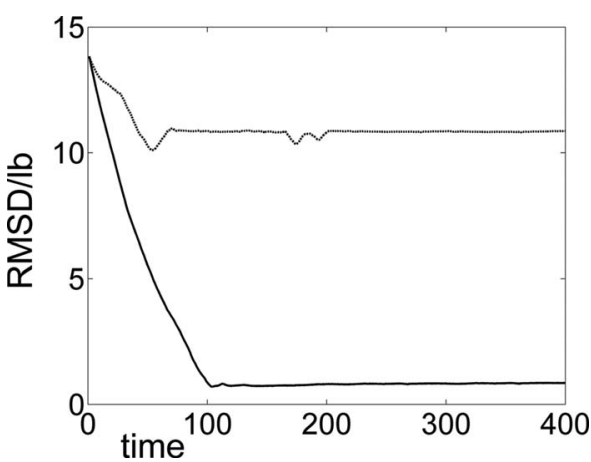

FIG. 2. rmsd values for the chain with all native contacts (solid line) and with only short-range native contacts (-.-.).

to be independent of time becomes shorter, as seen from the dashed curve of Fig. 2. The secondary structures form faster, and that the presence of long-range forces slows down the folding process. In conclusion, secondary structures form first, followed by their slow rearrangements in space to form the tertiary native state. These observations are consistent with experimental results. ${ }^{24}$

Figure 3 shows the time evolution of rmsd for secondary structures and the chain. It is obvious from the figure that individual helices form earlier than the whole chain. Figure 4 shows the average formation time with respect to the topological distance between two residues. We define the formation time as the first time the rmsd takes its final value. The fastest formation occurs when the residues are closest (two residues apart). When the residue index differences are 3-5, the formation times are close to each other. However, the formation time increases fast when the pairs are more than five residues apart. The time for long-range contacts is calculated as an average time for all pairs with $|i-j|>5$ and shown at the right hand side of the abscissa as long range. This plot also shows us that local structures form first, and the long-range contacts form during the later stages of folding, indicating that folding is hierarchical, supporting earlier observations. ${ }^{3,28,29}$

\section{B. Analysis of sequence of events during the folding process}

Analysis of our simulations has revealed that protein folding goes through some distinct phases. The first phase is the initial formation phase where the structure starts to resemble the native structure, the second phase is the restructuring phase in which some of the structures go through changes in their configurations, and the last phase is the final

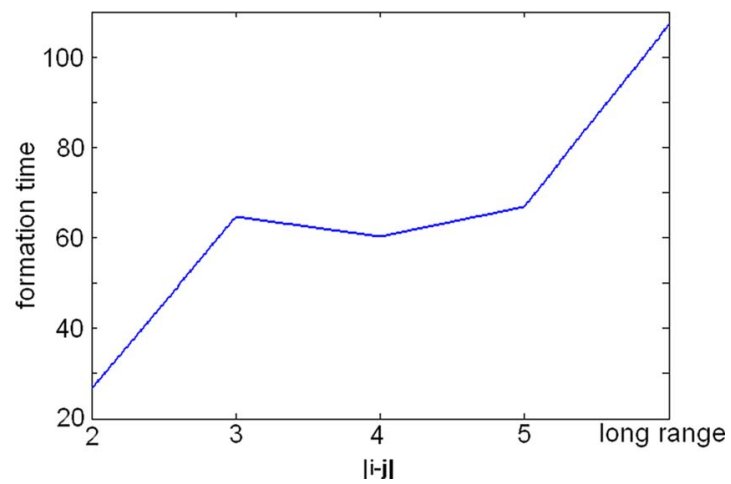

FIG. 4. Average formation time for 19 initial conditions with respect to residue index differences $2-5$, and long-range contact pairs.

formation phase where the protein settles into its final configuration. In this part, we try to clarify the sequence of such events by studying them in terms of the first formation time, deformation period and settling time. The first formation time is the first time the rmsd of a secondary structure takes on its final value. The deformation period is the time interval in which the rmsd values exceed the final rmsd value by a certain margin. Here the margin is taken to be $5 \%$ of the difference of initial and final rmsd values. The settling time is the time it takes for rmsd values to reach and stay within a neighborhood of the final rmsd value. This neighborhood is also defined to be $5 \%$ of the difference of initial and final rmsd values.

It can be seen from Fig. 3(a) that the formation time of helix 2, for example, is 23 , which is the end of the sudden decrease of the rmsd and the first time the rmsd value is within $5 \%$ of the difference between its initial and final rmsd values. Between times $28-55$, the rmsd values temporarily increase and stay outside the $5 \%$ limit. This period is termed the deformation period as the structure has "deformed." After time 55, the rmsd values enter between the 5\% limit again and remain always close to the final value, so the settling time for helix 2 is 55 .

In Table IV, we can see the first forming times of three helices, loop, and the turn in 1 vii. It can be observed that helix 2 forms first for 15 out of 19 initial conditions. As we look at the average forming times, we see that helix 2 is the fastest forming structure. The same conclusion appears in Duan and Kollman's study on villin headpiece ${ }^{4}$ which reports that the second helix is an initiation site for folding. Helix 2 with three contacts is the smallest structure in the (a)

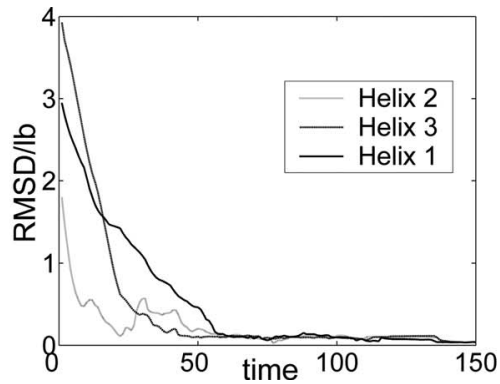

(b)

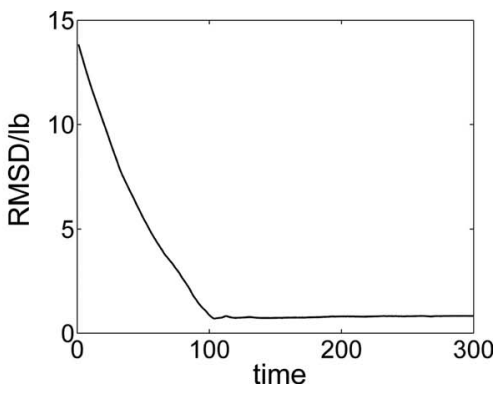

FIG. 3. (a) rmsd changes for the individual helices and (b) rmsd change for whole chain. $l_{b}$ : bond length distance (3.8 ̊). 
TABLE IV. First formation times for 19 different initial conditions.

\begin{tabular}{|c|c|c|c|c|c|}
\hline \multirow[b]{2}{*}{$\begin{array}{c}\text { Trajectory } \\
\text { No. }\end{array}$} & \multicolumn{5}{|c|}{ Times of first formation } \\
\hline & Helix 1 & Helix 2 & Helix 3 & Loop & Turn \\
\hline 1 & 58 & 18 & 61 & 101 & 62 \\
\hline 2 & 82 & 26 & 59 & 53 & 110 \\
\hline 3 & 84 & 24 & 79 & 23 & 98 \\
\hline 4 & 92 & 35 & 70 & 216 & 83 \\
\hline 5 & 85 & 56 & 62 & 226 & 77 \\
\hline 6 & 73 & 18 & 48 & 32 & 20 \\
\hline 7 & 86 & 15 & 60 & 24 & 25 \\
\hline 8 & 47 & 45 & 51 & 40 & 60 \\
\hline 9 & 41 & 43 & 76 & 50 & 85 \\
\hline 10 & 55 & 58 & 67 & 70 & 81 \\
\hline 11 & 75 & 22 & 44 & 102 & 76 \\
\hline 12 & 52 & 48 & 76 & 84 & 55 \\
\hline 13 & 88 & 34 & 70 & 17 & 21 \\
\hline 14 & 72 & 69 & 69 & 74 & 52 \\
\hline 15 & 90 & 87 & 71 & 165 & 54 \\
\hline 16 & 63 & 43 & 51 & 31 & 26 \\
\hline 17 & 30 & 19 & 38 & 38 & 13 \\
\hline 18 & 40 & 58 & 37 & 19 & 28 \\
\hline 19 & 48 & 23 & 27 & 13 & 65 \\
\hline Average & 66.36 & 39 & 58.73 & 72.52 & 57.42 \\
\hline Std dev. & 19.07 & 19.46 & 14.44 & 61.63 & 27.95 \\
\hline
\end{tabular}

protein. Our optimization tries to bring the contact distances to their native values. Since the number of contacts that define the formation of helix 2 is small, optimization finds it easiest to form this smallest structure first. The loop and the turn have the highest standard deviations of rmsds, indicating that their first formation times are more dependent on initial condition than the helices.

In Table $\mathrm{V}$ the deformation time intervals are tabulated for different structures. Deformation time intervals correspond to the intervals in which rmsd values increase and stay above the final rmsd value by a certain margin. Here the margin is taken to be $5 \%$ of the difference of initial and final rmsd values. Table V shows that helix 2, which was found to be the easiest one to form, also has the largest number of deformations for most of the initial conditions. This observation is in agreement with molecular dynamics simulation results where helix 2 was observed to be partially formed in 12 of the 13 snapshots throughout the simulations. ${ }^{4}$ On the other hand, helices 1 and 3 are more stable once they form. Helix 3 is stable for all initial conditions and helix 1 goes through reconstruction for four initial conditions. In our model, there are 6,3 , and 15 native contacts defined for helices 1,2 , and 3 , respectively. Thus, the energy contribution of helices 1 and 3 into the objective function is greater than that of helix 2. Consequently, optimization tries to keep helix 1 and especially helix 3 more stable by not allowing them to go through unnecessary deformation. Helix 2 is between the loops and other helices. Therefore, it plays a damping role between these structures. It moves freely to achieve the tight regulation of the formation of helices 1 and 3 . As a result it undergoes more reconstructions to help the formation of the more demanding helices 1 and 3 .

Table VI shows the final formation dynamics by giving
TABLE V. Deformation time intervals for three helices for 19 initial conditions.

\begin{tabular}{|c|c|c|c|}
\hline \multirow{2}{*}{$\begin{array}{c}\text { Trajectory } \\
\text { No. }\end{array}$} & \multicolumn{3}{|c|}{ Deformation time intervals } \\
\hline & Helix 1 & Helix 2 & Helix 3 \\
\hline 1 & $(62-87)$ & $(52-67),(72-101),(152-183)$, & $\cdots$ \\
\hline 2 & $\cdots$ & $27-51$ & $\cdots$ \\
\hline 3 & $\cdots$ & $25-70 / 99-111$ & $\cdots$ \\
\hline 4 & $280-298$ & $\cdots$ & $\cdots$ \\
\hline 5 & $86-133$ & $(107-118),(126-131),(188-225)$ & $\cdots$ \\
\hline 6 & $\cdots$ & $30-68$ & $\cdots$ \\
\hline 7 & $\cdots$ & $240-256$ & $\cdots$ \\
\hline 8 & $\cdots$ & $\cdots$ & $\cdots$ \\
\hline 9 & $\cdots$ & $59-95$ & $\cdots$ \\
\hline 10 & $\cdots$ & $(100-136),(167-216)$ & $\cdots$ \\
\hline 11 & $\cdots$ & $23-54$ & $\cdots$ \\
\hline 12 & $\cdots$ & $255-266$ & $\cdots$ \\
\hline 13 & $144-169$ & $176-188$ & $\cdots$ \\
\hline 14 & $\cdots$ & $\cdots$ & $\cdots$ \\
\hline 15 & $\cdots$ & $145-159$ & $\cdots$ \\
\hline 16 & $\cdots$ & $62-71$ & $\cdots$ \\
\hline 17 & $\cdots$ & $\cdots$ & $\cdots$ \\
\hline 18 & $\cdots$ & $\cdots$ & $\cdots$ \\
\hline 19 & $\cdots$ & $\cdots$ & $\cdots$ \\
\hline
\end{tabular}

the settling times (i.e., times at which the structures reach and stay within $5 \%$ of the difference of their initial and final rmsd values). The settling times which are higher than 250 are indicated with stars. These are the structures which cannot settle into a final state within the allowed time of 301 time steps. Based on the computed average values, the loop and the turn settle last. In fact, for some initial conditions,

TABLE VI. Settling times. The numbers higher than 250 are indicated with stars.

\begin{tabular}{|c|c|c|c|c|c|}
\hline \multirow{2}{*}{$\begin{array}{c}\text { Trajectory } \\
\text { No. }\end{array}$} & \multicolumn{5}{|c|}{ Settling times } \\
\hline & Helix 1 & Helix 2 & Helix 3 & Loop & Turn \\
\hline 1 & 87 & 183 & 61 & 209 & 194 \\
\hline 2 & 82 & 51 & 59 & 95 & 110 \\
\hline 3 & 84 & 111 & 79 & $278^{*}$ & 117 \\
\hline 4 & $298^{*}$ & $268^{*}$ & 70 & $272^{*}$ & 240 \\
\hline 5 & 133 & 226 & 62 & 226 & 225 \\
\hline 6 & 73 & 68 & 48 & $290^{*}$ & $293^{*}$ \\
\hline 7 & 86 & $256^{*}$ & 60 & $299^{*}$ & 187 \\
\hline 8 & 47 & 45 & 51 & 207 & 195 \\
\hline 9 & 41 & 95 & 76 & 184 & 85 \\
\hline 10 & 55 & 215 & 67 & 251 & 237 \\
\hline 11 & 75 & 54 & 44 & 102 & 78 \\
\hline 12 & 52 & $266^{*}$ & 76 & $265^{*}$ & 278 \\
\hline 13 & 169 & 188 & 70 & 200 & 200 \\
\hline 14 & 72 & 69 & 68 & 93 & 87 \\
\hline 15 & 90 & 159 & 71 & 165 & 202 \\
\hline 16 & 63 & 71 & 51 & $283^{*}$ & $288^{*}$ \\
\hline 17 & 30 & 19 & 38 & 120 & 101 \\
\hline 18 & 40 & $284^{*}$ & 37 & $285^{*}$ & $292^{*}$ \\
\hline 19 & 48 & 23 & 27 & 142 & 32 \\
\hline Average & 85.52 & 139.52 & 58.68 & 208.73 & 181.10 \\
\hline
\end{tabular}



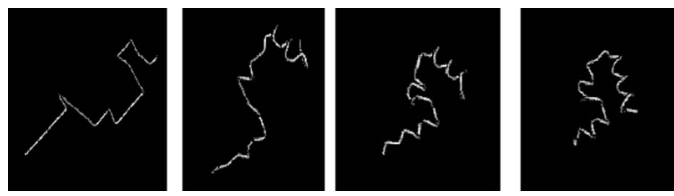

FIG. 5. Snapshots from the folding process starting from an arbitrary initial condition. They show the configurations at times $0,25,50$, and 75 from left to right.

the loop and the turn are not able to settle to their final values (these are indicated with a star). Helix 3 settles first. Since helix 3 is costly to form in terms of energy, it is stable once it forms; thus, it has the shortest settling time.

In Fig. 5 the progress of conformations throughout time can be observed. The first picture shows the initial configuration of the chain. The second shows the formation of helices. In the third picture almost all helices are formed. In the last picture, the correct orientations of secondary structures are reached with the help of attractive forces between the long-range contact pairs.

The simulations do not provide absolute time scales, but relative ones, after rescaling the duration (number of time steps) of particular events with respect to experimental data. Such time scales for the sequence of events observed in our simulations are assigned here by comparing with the experimental data of Kubelka et al. ${ }^{24}$ Experiments show that helix formation time in the villin is around $70 \mathrm{~ns}$. Simulations of Duan and Kollman ${ }^{4}$ show that native helical content is around $60 \%$ at the end of $100 \mathrm{~ns}$ and show a steady increase after $200 \mathrm{~ns}$. Earlier time resolved infrared spectroscopy showed that the formation of a 21-residue alanine based peptide is about $200 \mathrm{~ns}^{30}$ So, a value in the range of 100-200 ns may be assigned to the stable formation of helices, which is consistent with earlier estimates. ${ }^{1}$ The most stable helix in the chicken villin headpiece is helix 3, with a formation time of 58.68 computational time units reported in Table VI. Comparing this value with the 100-200 ns range of real time shows that one computational time unit in our simulations corresponds to approximately $2-4 \mathrm{~ns}$ of real time. Based on this estimate, we find from the last row of Table VI that the average time for the loop to form lies in the range of $418-836 \mathrm{~ns}$ and the average time for the turn to form is in the range of $362-714$ ns. Temperature jump experiments show the formation of turns to be around $500 \mathrm{~ns}$, which is within the range of our calculations. ${ }^{1}$ The event that determines the longest folding time in our calculations is the formation of the loops, followed by the overall rearrangements of the secondary structure units. These rearrangements are small, however, and terminate around 300 computation time scales. In the real time scale, this corresponds to a range of $600 \mathrm{~ns}-1.2 \mu \mathrm{s}$. This range corresponds to the overall folding time in our calculations and is smaller than the experimental ${ }^{24}$ and simulation results obtained by the Folding at Home Project ${ }^{26}$ that give an overall folding time of 4-5 $\mu \mathrm{s}$. The optimal folding times that we obtain may be accepted as a lower bound since the computational scheme searches always for the fastest routes to folding on the energy landscape.

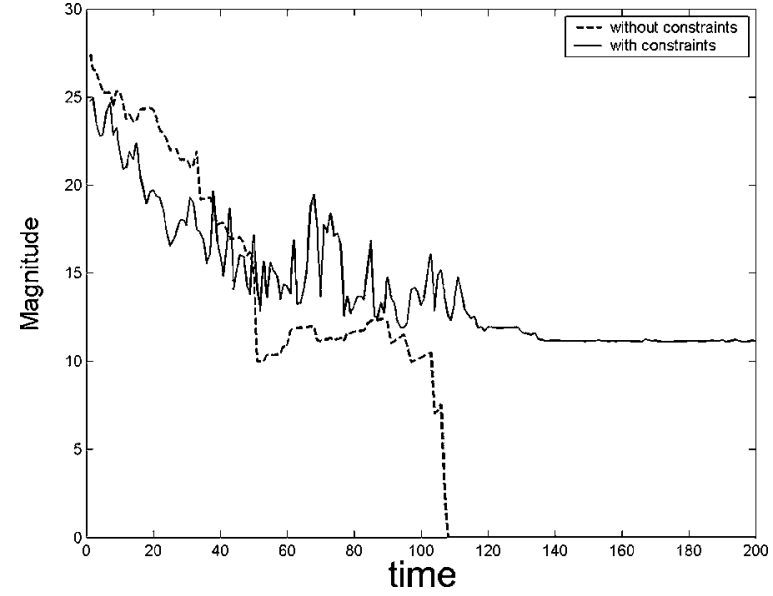

FIG. 6. Effect of excluded volume and bond length constraints on the optimal force magnitudes.

\section{Analysis of the force field}

The optimal force field calculated by the optimization is responsible for (i) bringing the native contact pairs together, (ii) providing the repulsive effects on the beads when they tend to get closer than their allowed distance (excluded volume effect), and (iii) keeping the bond length between bonded beads within its allowed limits. Here we will analyze this force field using different tools to gain additional insight into the folding dynamics.

In order to isolate and analyze the different components of the force field, we folded the protein from its first initial condition (trajectory 1) by first including all the bond length and excluded volume constraints and next without these constraints. We next compared the resulting magnitudes of forces acting on the secondary structures. Each optimal force applied on a bead has its $x, y$, and $z$ components in three dimensions, $u_{i}(x), u_{i}(y)$, and $u_{i}(z)$, respectively. Total force acting on a particular structure can be easily computed by adding the magnitudes of the forces acting on the individual beads of that structure. For example, Fig. 6 compares the forces acting on helix 3 for the two optimizations. When bond length and excluded volume constraints are absent, the whole chain collapses to a point as expected under purely attractive forces. In the absence of bond length or excluded volume constraints, the force field does not exhibit any significant high frequency behavior. However, when optimization includes all the constraints, Fig. 6 shows that the force field has to make finer adjustments with higher frequency content to find a feasible folding pathway in an environment that is severely restricted by many bond length and excluded volume constraints ( 35 bond length constraints and 595 excluded volume constraints).

Figure 7 shows the behavior of energy under the two force fields. When constraints are not present, the force field brings the energy quickly down to its least attainable value of zero. But in the presence of bond length and excluded volume restrictions, the energy decays more slowly to its final optimal value.

Using Eq. (31) we reconstructed the optimal force components by different number of modes and applied these reconstructed forces to the protein. The resulting energy values 


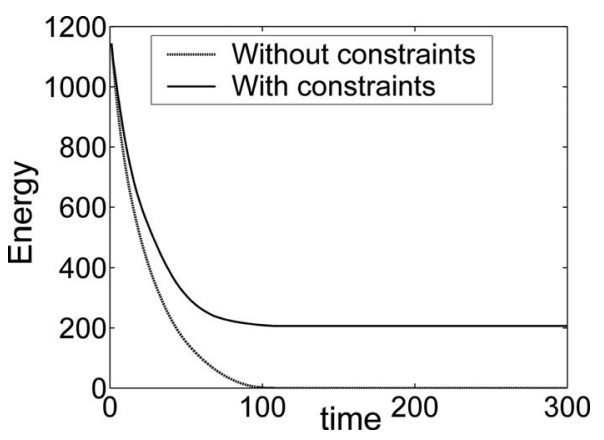

FIG. 7. Optimal energy change with and without constraints.

are computed and presented in Fig. 8(a). The first mode contributes to most of the energy minimization by decreasing the energy monotonically to its final value. The addition of the second mode speeds up the folding process significantly, as energy drops more quickly. Native contacts are brought together faster with more attractive forces resulting with the additional mode. It is seen that higher modes (i.e., mode 3 and above) do not have significant contributions to the energy as the energy curve with two modes is already very close to the optimal energy curve. A similar trend is observed in rmsd behavior, as shown in Fig. 8(b). As with energy change, the final rmsd value is reached much faster with the addition of higher modes. However, in the case of rmsd changes the contribution of the higher modes (i.e., above three modes) should not be disregarded. Figure 8(b) shows that addition of higher modes (as many as 30 modes are shown) speeds up the response of rmsd values.

Next, we analyze the contribution of different structures to the total energy. Each structure's contribution consists of the attractive energies of the native contacts within that structure (short range) and the energies due to its contacts with other structures (long range). These two sets of energies are plotted in Figs. 9 and 10, respectively.

Figure 9 shows that local energies of helix 2 and especially the tail at the C-terminal (residues 31-36) increase at certain times so that higher energy contents of other structures and thus the total energy can be better minimized. However, Fig. 10 shows that the tail's energy due to its longrange native contacts with other structures in the chain is dominating and thus optimization chooses not to sacrifice it

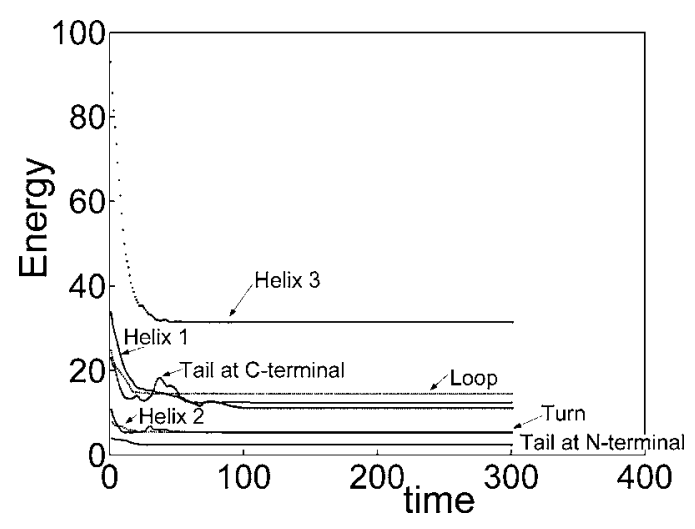

FIG. 9. Energy contributions due to short-range contacts of individual secondary structures of the protein.

and decreases it continuously. In fact, all energies resulting from interstructure interactions show a monotonic decrease. In conclusion, the energy of the protein is distributed among its structures during folding, and how this distribution changes over time depends on how native contacts are distributed among the structures.

Bond length and excluded volume constraints are major hindrances to the folding of proteins. Although higher modes of the force field are less important for energy minimization as we have seen in the above analysis, they are the ones that maintain the constraints feasible. In order to demonstrate this fact, reconstructed optimal forces using different number of modes were applied to the protein and its folding was compared. Figures 11(a) and 11(b) show the results. The bond lengths have to be between $0.95 l_{b}$ and $1.05 l_{b}$ in our optimization, where $l_{b}=3.8 \AA$. Therefore Fig. 11(a) shows that the particular bond length is violated most of the time for one or three modes. The number of violations decreases as one adds more modes to the force field and it takes more than 30 modes to attain feasibility with respect to this particular bond length.

Similarly, Fig. 11(b) shows the behavior of the excluded volume between beads 7 in helix 1 and 10 in loop. The excluded volume constraint is violated at some time during folding if higher modes are not used. It takes more than 30 modes to achieve feasibility for this excluded volume at all times. In conclusion, it is shown that higher modes of the (a)

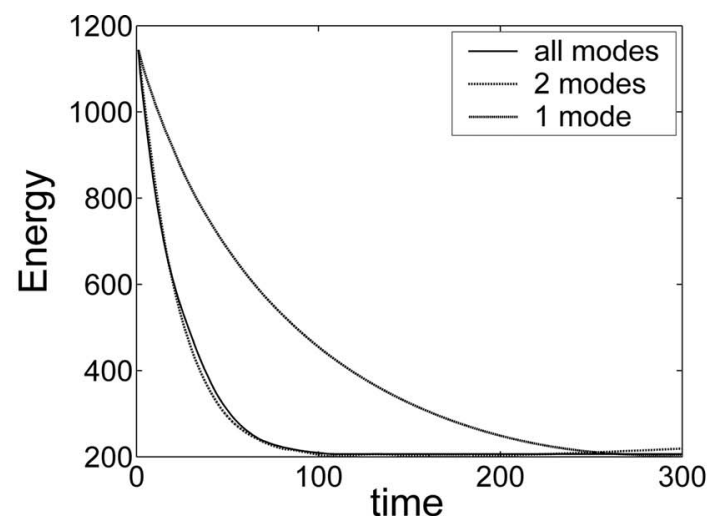

(b)

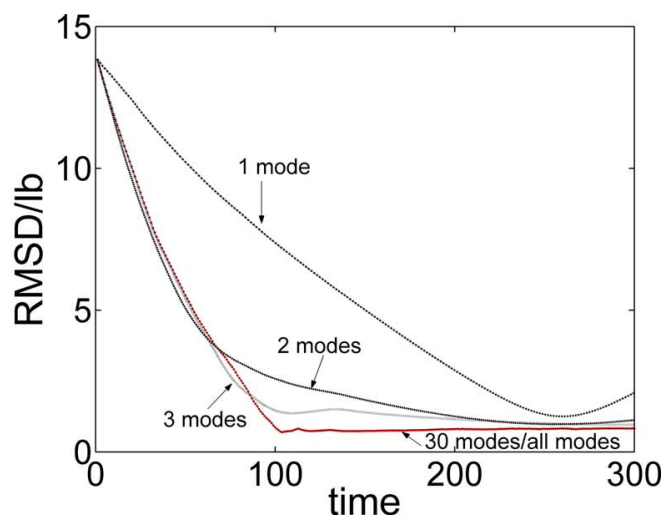

FIG. 8. (a) Energy change with optimal (all modes) and reconstructed forces by 1 and 2 modes. (b) rmsd change under the optimal force (all modes) and under the forces reconstructed by $1,2,3$, and 30 modes. 


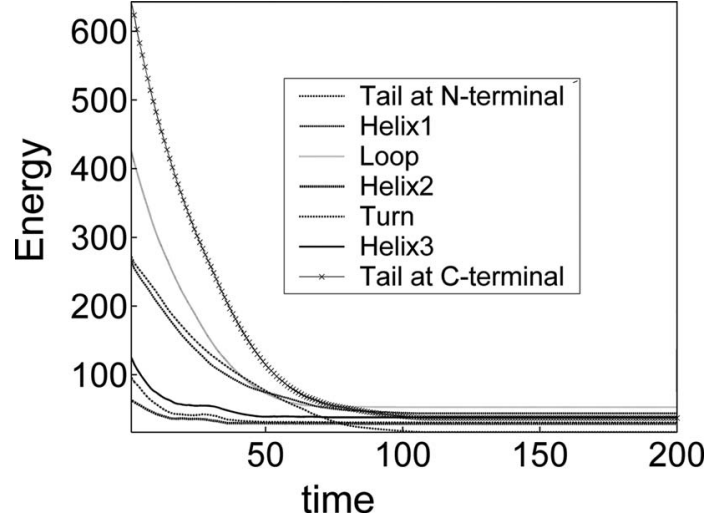

FIG. 10. Energy contributions due to long-range contacts between different secondary structures.

force field (or high frequency forces) are required for satisfying both bond length and excluded volume constraints. This is consistent with Fig. 6 where we have seen that the force field has to make higher frequency adjustments to satisfy the constraints.

\section{CONCLUSION}

The folding of a denatured protein to its unique threedimensional folded native state is a complex problem, which has attracted a great deal of interest in recent years. Despite numerous theoretical and experimental studies, a comprehensive understanding of many aspects of the protein folding is still lacking. ${ }^{31}$

Various pathways are available to a folding protein that start from a random initial configuration and end in the native state. Each path is determined by the succession of selfinteractions of the elements that make up the protein molecule. ${ }^{29}$ Pathways can be described in terms of sequence of events. Formations of secondary structures, deformations of a helix, or entrance of a loop between two structures are few examples of such events. ${ }^{29}$ Computer simulations can provide rich information about pathways. In this regard, we introduced a new dynamic optimal control approach based on a coarse-grained model in which successive residues along the chain are taken as linked beads. We assume that native structure of the target protein is known and we obtain

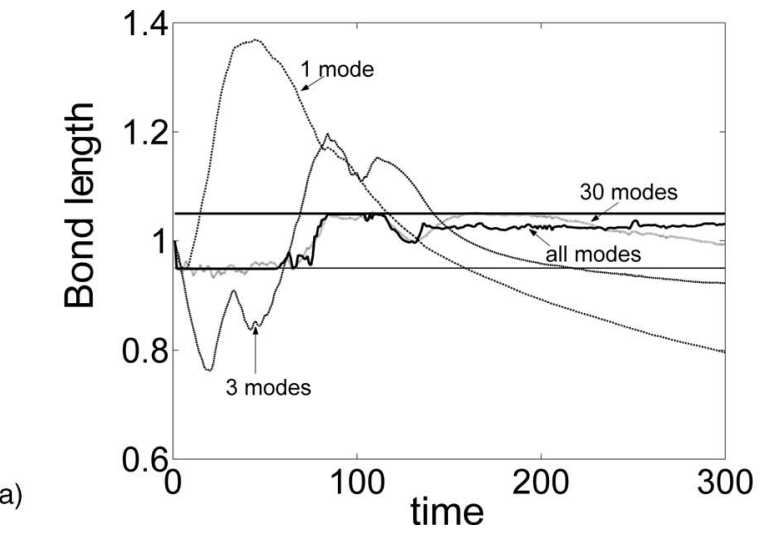

the native contact data from the native structure. In order to generate the linear connectivity of the protein, we model the interaction between covalently bonded beads as linear springs. The other interactions are defined as a force field that helps to fold the protein from an initial condition to a final native state in a feasible (i.e., without violating excluded volume and bond length constraints) and an optimal way (i.e., with respect to the defined objective function). In our approach, these forces are automatically computed to deliver the desired folding. We studied a small, fast folding protein, chicken villin headpiece. It has 36 residues that form three short helices, a loop, and a turn.

We obtained 19 pathways starting from random initial conditions. We studied the sequence of events for each pathway. This sequence of events includes formation of helices, loops, and turns, deformation periods of these substructures, and settling times for each structure. We observed that there is no unique pathway for proteins starting from random initial conditions. Each pathway has its own succession of events. However, we also observed some common characteristics for most of the pathways. It has been observed that the helix 2 is the fastest forming structure in folding of villin headpiece for different pathways. The result is in accordance with the molecular dynamics (MD) simulations of villin by Duan and Kollman. ${ }^{4}$ Long-range and short-range contacts and their formation are also studied. We found that shortrange contacts which are responsible for the formation of helices and orientation of successive substructures are formed prior to the long-range contacts. Long-range contacts are the tertiary contacts and they provide the orientation of secondary structures in three-dimensional (3D) space. This result also conforms to the finding that tertiary contacts are less likely to form in the early stages of folding. ${ }^{4}$

Lastly, we tried to analyze the force field we obtained from the optimization program using a particular type of principal component analysis known as KLE. Force field trajectories are decomposed into modes. It is observed that high frequency behavior of the force field is mainly due to the repulsive forces that try to satisfy excluded volume constraints and to prevent the covalent bonds to deviate from the assigned value, $3.8 \AA$. On the other hand, energy of the protein (minimum attractive energies between nonbonded native contact pairs) can be represented quite well with the result of

(b)

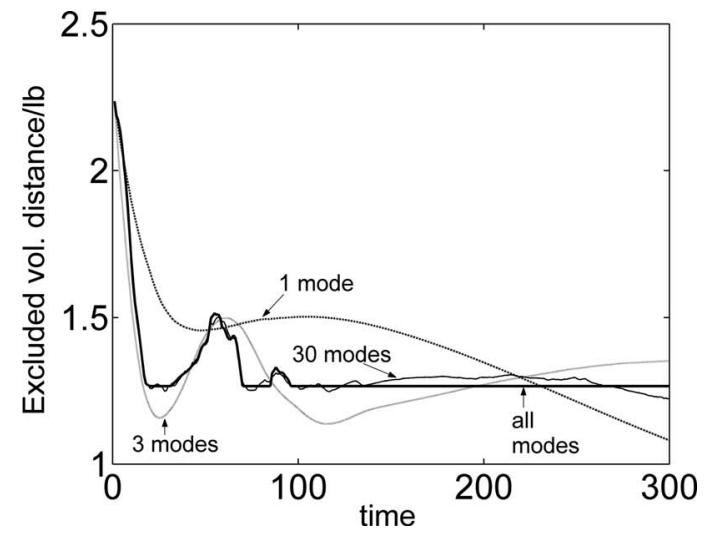

FIG. 11. (a) Dependence of bond length fluctuations on the number of modes used in calculations. (b) Change of the excluded volume distance between beads 7 and 10. 
simulations with the few modes of force field. Therefore, we deduced that first few modes of the force field could explain the attractive forces between nonbonded pairs, and higher modes capture more of the higher frequency repulsive forces.

Our method is a new approach and it is easy to construct and implement. Since it is a simplified model, it lacks the molecular details that MD simulations include. However, we can generate feasible and optimal pathways by the machinery of the proposed optimal control formulation and use these pathways to get further insight into folding. In particular, we can extract valuable information about general characteristics of optimal folding pathways and sequence of events and properties of force fields acting on molecules. Besides, we have the flexibility to change and improve an objective function, i.e., energy definition of the system.

Recently, Carr and Wales ${ }^{27}$ studied the folding pathways of the villin headpiece using a Monte Carlo minimization method, where every step in the simulation is followed by local minimization of the resulting configuration. They used the unified residue model ${ }^{32}$ and the related potentials that are more detailed than that of the present study. As future work, the unified residue model will be implemented into our dynamic optimization model and interest will be focused on predictive nature of the computations as was the case in the work of Carr and Wales. ${ }^{27}$ Modifications in the dynamic model and objective function along this direction and improvements in computation time are currently under investigation in our group.

\section{ACKNOWLEDGMENTS}

We want to acknowledge the funding received from TÜBİTAK through Grant No. 104E004. We also want to thank Serife Senturk for the help she provided with some of the simulations. We thank Professor Lorenz T. Biegler of Carnegie Mellon University for his helpful comments on optimization.

\footnotetext{
${ }^{1}$ W. A. Eaton, V. Munoz, P. A. Thompson, C. K. Chan, and J. Hofrichter, Curr. Opin. Struct. Biol. 7, 10 (1997).

${ }^{2}$ R. H. Callender, R. B. Dyer, R. Gilmanshin, and W. H. Woodruff, Annu. Rev. Phys. Chem. 49, 173 (1998); M. Gruebele, ibid. 50, 485 (1999); W. A. Eaton, V. Munoz, S. J. Hagen, G. S. Jas, L. J. Lapidus, E. R. Henry, and J. Hofrichter, Annu. Rev. Biophys. Biomol. Struct. 29, 327 (2000); J. K. Myers and T. G. Oas, Annu. Rev. Biochem. 71, 783 (2002); J. Kubelka, J. Hofrichter, and W. A. Eaton, Curr. Opin. Struct. Biol. 14, 76 (2004).

${ }^{3}$ M. Cieplak and T. X. Hoang, Biophys. J. 84, 475 (2003).

${ }^{4}$ Y. Duan and P. A. Kollman, Science 282, 740 (1998).

${ }^{5}$ M. Vendruscolo, R. Najmanovich, and D. Domany, Phys. Rev. Lett. 82, 656 (1999); P. Doruker, R. L. Jernigan, and I. Bahar, J. Comput. Chem. 23, 119 (2002); T. Haliloglu and I. Bahar, Proteins: Struct., Funct., Genet. 31, 271 (1998).

${ }^{6}$ A. A. Palazoglu, A. Gursoy, Y. Arkun, and B. Erman, J. Comput. Biol. 11, 1149 (2004)

${ }^{7}$ P. J. Flory, Statistical Mechanics of Chain Molecules (Wiley, New York, 1969).

${ }^{8}$ A. Bejan, Advanced Engineering Thermodynamics, 2nd ed. (Wiley Inter-
}

science, New York, 1997); A. Bejan, J. Adv. Transp. 30, 85 (1996).

${ }^{9}$ B. Erman and K. A. Dill, J. Chem. Phys. 112, 1050 (2000).

${ }^{10}$ S. Miyazawa and R. L. Jernigan, J. Mol. Biol. 256, 623 (1996); I. Bahar and R. L. Jernigan, Folding Des. 1, 357 (1996).

${ }^{11}$ C. Clementi, A. Maritan, and J. R. Banavar, Phys. Rev. Lett. 81, 3287 (1998); R. I. Dima, G. Settanni, and C. Micheletti, J. Chem. Phys. 112, 9151 (2000); F. Seno, C. Micheletti, A. Maritan, and J. R. Banavar, Phys. Rev. Lett. 81, 2172 (1998); C. Micheletti, A. Maritan, and J. R. Banavar, J. Chem. Phys. 110, 9730 (1999); C. Micheletti, F. Seno, and J. R. Banavar, Proteins 42, 422 (2001); J. van Mourik, C. Clementi, and A. Maritan, J. Chem. Phys. 110, 10123 (1999); A. Erkip, B. Erman, C. Seok, and K. A. Dill, Polymer 43, 495 (2001); P. D. Thomas and K. A. Dill, J. Mol. Biol. 257, 457 (1996).

${ }^{12}$ J. W. Ponder and D. A. Case, Adv. Protein Chem. 66, 27 (2003).

${ }^{13}$ D. Roccatano, I. Daidone, M. Ceruso, C. Bossa, and A. DiNolay, Biophys. J. 84, 1876 (2003); S. A. J. Allen, A. R. Curran, R. H. Templer, W. Meijberg, and P. J. Booth, J. Mol. Biol. 342, 1293 (2004); F. Khan, J. I. Chuang, S. Gianni, and A. R. Fersht, ibid. 333, 169 (2003); T. Pan, X. Fang, and T. Sosnick, ibid. 286, 721 (1999); M. Jacob, T. Schindler, J. Balbach, and F. X. Schmid, Proc. Natl. Acad. Sci. U.S.A. 94, 5622 (1997); Z. D. Su, M. T. Arooz, H. M. Chen, C. J. Gross, and T. Y. Tsong, ibid. 93, 2539 (1996); K. Plaxco and D. Baker, ibid. 95, 13591 (1998); D. Baurecht, I. Porth, and U. P. Fringeli, Vib. Spectrosc. 30, 85 (2002); J. H. Cho, S. Sato, and D. P. Raleigh, J. Mol. Biol. 338, 827 (2004); G. C. Brown, H. V. Westerhoff, and B. N. Kholodenko, J. Mol. Biol. 182, 389 (1996); U. Shinde and M. Inouye, Semin Cell Dev. Biol. 11, 35 (2000).

${ }^{14}$ I. Bahar, M. Kaplan, and R. L. Jernigan, Proteins 29, 292 (1997).

${ }^{15}$ T. Haliloglu, I. Bahar, and B. Erman, Phys. Rev. Lett. 79, 3090 (1997).

${ }^{16}$ A. E. Bryson and Y. C. Ho, Applied Optimal Control (Taylor \& Francis, New York, 1975)

${ }^{17}$ L. T. Biegler, A. M. Cervantes, and A. Waetcher, Chem. Eng. Sci. 57, 575 (2002).

${ }^{18}$ U. M. Ascher and L. R. Petzold, Computer Methods for Ordinary Differential Equations and Differential-Algebraic Equations (SIAM, Philadelphia, PA, 1998).

${ }^{19}$ A. Ben-Tal and M. Zibulevsky, SIAM J. Optim. 7, 347 (1997).

${ }^{20}$ M. Kocvara and M. Stingl, PENnon edited by G. D. P. a. A. Murli, 2003, p. 297.

${ }^{21}$ A. Mukherjee and B. Bagchi, J. Chem. Phys. 118, 4733 (2003); D. Spoel and E. Lindahl, J. Phys. Chem. B 107, 11178 (2003); A. Fernandez, M. Shen, A. Colubri, T. R. Sosnick, R. S. Berry, and K. F. Freed, Biochemistry 42, 664 (2003); T. Herges and W. Wenzel, Biophys. J. 87, 3100 (2004); G. M. S. Mori, C. Micheletti, and G. Colombo, J. Phys. Chem. B 108, 12267 (2004).

${ }^{22}$ Y. Tang, D. J. Rigotti, R. Fairman, and D. P. Raleigh, Biochemistry 43, 3264 (2004).

${ }^{23}$ M. Wang, Y. Tang, S. Sato, L. Vugmeyster, C. J. McKnight, and D. P. Raleigh, J. Am. Chem. Soc. 125, 6032 (2003).

${ }^{24}$ J. Kubelka, W. A. Eaton, and J. Hofrichter, J. Mol. Biol. 329, 625 (2003).

${ }^{25}$ G. M. S. Mori, G. Colombo, and C. Micheletti, Proteins: Struct., Funct., Bioinf. 58, 459 (2005).

${ }^{26}$ B. Zagrovic, C. D. Snow, M. R. Shirts, and V. J. Pande, J. Mol. Biol. 323, 927 (2002).

${ }^{27}$ J. M. Carr and D. J. Wales, J. Chem. Phys. 123, 234901 (2005).

${ }^{28}$ D. Baker, Nature (London) 405, 39 (2000); R. L. Baldwin and G. D. Rose, Trends Biochem. Sci. 24, 26 (1999).

${ }^{29}$ B. Erman, Biophys. J. 81, 3534 (2001).

${ }^{30}$ K. Williams, T. P. Causgrove, R. Gilmanshin, K. S. Fang, R. H. Callender, W. H. Woodruff, and R. B. Dyer, Biochemistry 35, 691 (1996).

${ }^{31}$ G. Srinivas and B. Bagchi, J. Phys. Chem. B 107, 11768 (2003).

${ }^{32}$ A. Liwo, S. Ołdziej, M. R. Pincus, R. J. Wawak, S. Rackovsky, and H. A. Scheraga, J. Comput. Chem. 18, 849 (1997); A. Liwo, M. R. Pincus, R. J. Wawak, S. Rackovsky, S. Ołdziej, and H. A. Scheraga, ibid. 18, 874 (1997); A. Liwo, R. Kaźmierkiewicz, C. Czaplewski, M. Groth, S. Oldziej, R. J. Wawak, S. Rackovsky, M. R. Pincus, and H. A. Scheraga, ibid. 19, 259 (1998). 